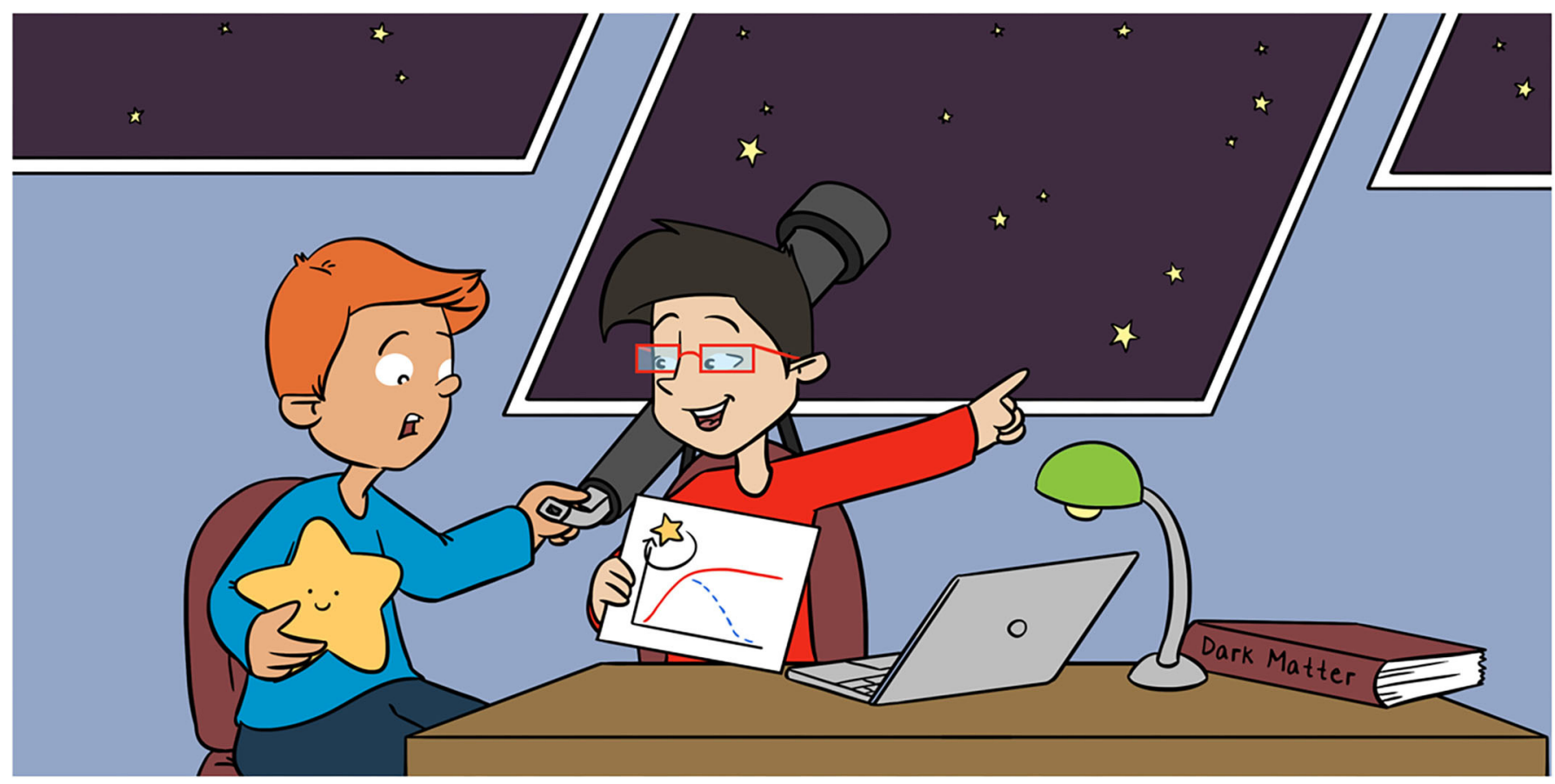

\title{
HOW DO SCIENTISTS KNOW DARK MATTER EXISTS?
}

Vishnu Prithiv Bhathe ${ }^{1}$, Christina Brennan ${ }^{1}$, Stephanie Ellis ${ }^{1}$, Emily Moynes ${ }^{1}$, Kevin Graham ${ }^{2}$ and Sean J. Landsman ${ }^{1^{*}}$

${ }^{1}$ Institute of Environmental and Interdisciplinary Science, Carleton University, Ottawa, ON, Canada

${ }^{2}$ Department of Physics, Carleton University, Ottawa, ON, Canada

\section{YOUNG REVIEWERS:}

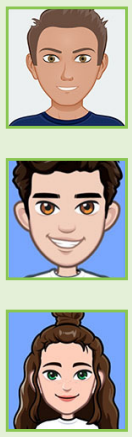

SANAH AGE: 14

There is still a lot we do not know about the universe. Understanding the existence and make-up of a mysterious substance called dark matter is one of the leading challenges scientists face today. There are many theories about what dark matter could be, but we have yet to understand its true nature. How do we even know that such a thing exists? The greatest challenge for studying dark matter is that we cannot see it. In this article, we will discuss how scientists use science and observations from telescopes to predict the existence of dark matter and why scientists think it pervades every corner of our universe.

\section{WHAT IS DARK MATTER? IS IT EVEN REAL?}

When we look up into the night sky, we notice that it is filled with thousands of stars. These bright celestial bodies include planets in our solar system, stars in our galaxy, and entire galaxies that are far, far away. These objects make up all the light-emitting matter in the 
ELECTRO-MAGNETIC SPECTRUM

The full range of light frequencies, from radio waves to gamma rays and X-rays.

\section{WAVELENGTH}

A measurement of light, specifically the distance between peaks in the light waves. Light wavelengths are measured in nanometers $(\mathrm{nm})$ and range from about $400 \mathrm{~nm}$ (ultraviolet) to $700 \mathrm{~nm}$ (infrared), with visible light falling in between.

MATTER

Anything that has mass

\section{DARK MATTER}

Matter that does not emit light and thus cannot be viewed with telescopes.

\section{ASTROPHYSICIST}

A scientist who studies astronomical objects, up to the size of the entire universe. universe. With the help of different kinds of telescopes, we can observe these celestial bodies through the light they radiate. Some telescopes can detect light from millions of light years away. In fact, one super telescope you may have heard of, the Hubble telescope, can see more than 13.4 billion light years away [1]! All telescopes work by detecting light in the electro-magnetic spectrum, from visible light to X-rays, emitted by these celestial bodies. Scientists use the various wavelengths of detected light to determine key information about our universe's celestial bodies, such as distance away, age, size, and shape. They can even use some of this information to understand the laws of the universe. Yet, there is matter in the universe that does not emit light in any part of the electro-magnetic spectrum, which means that we cannot observe it with our telescopes. This unique property makes it impossible to observe these types of matter, so scientists call it dark matter.

Some scientists, specifically astrophysicists, spend a great deal of time generating theories about what dark matter could be. Scientists know that dark matter does not emit light from any part of the electro-magnetic spectrum, but dark matter has been observed to be influenced by gravity. Astrophysicists are still unsure what dark matter is, exactly. However, they know what dark matter is not, by observing the way it behaves compared to other materials. We know that dark matter makes up $\sim 80 \%$ of the total mass of galaxies [2]. This means that there is four times more dark matter compared to regular matter! If dark matter is so difficult to observe, why do scientists believe it actually exists? The evidence to support the existence of dark matter is extensive, and we will explore three main examples in the following sections.

\section{DARK MATTER AFFECTS THE MOVEMENT OF STARS WITHIN GALAXIES}

The first type of evidence supporting the existence of dark matter has to do with the way dark matter affects the movement of celestial bodies. In our solar system, almost all of the mass is in the sun. The innermost planets like Mercury and Venus orbit the sun the fastest. As the distance from the sun increases, the speed at which planets move decreases. This is because there is less gravitational pull from the sun on planets farther out and, to keep from spiraling into or away from the sun, they must move slower. We can apply a similar analogy to galaxies. If we assume that the bright part of a galaxy shows where most of the mass is, then most of the mass is near the center, and at the dim edge of a galaxy there should not be much mass. Therefore, objects orbiting far from the center of the galaxy should move slower than objects closer to the center, just like the planets in our solar system.

To test this hypothesis, scientists recorded the incoming light from a distant spiral galaxy (our home galaxy, the Milky Way, is also considered 


\section{Figure 1}

The relationship between the velocity of stars and their distance from the center of the galaxy. The actual observed velocity of stars (A) that are far from the center of the galaxy is greater than what was predicted by scientists (B). This data indicates that there is a large amount of mass present in the outer part of the galaxy that we cannot see because it does not emit light, suggesting the presence of dark matter (Modified from original image by PhilHibbs and licensed under CC BY-SA 3.0).

\section{VELOCITY}

The distance moved between two points per unit of time. For example, a car moving $60 \mathrm{~km} / \mathrm{h}$ travels $60 \mathrm{~km}$ from Point A to Point B in $1 \mathrm{~h}$.

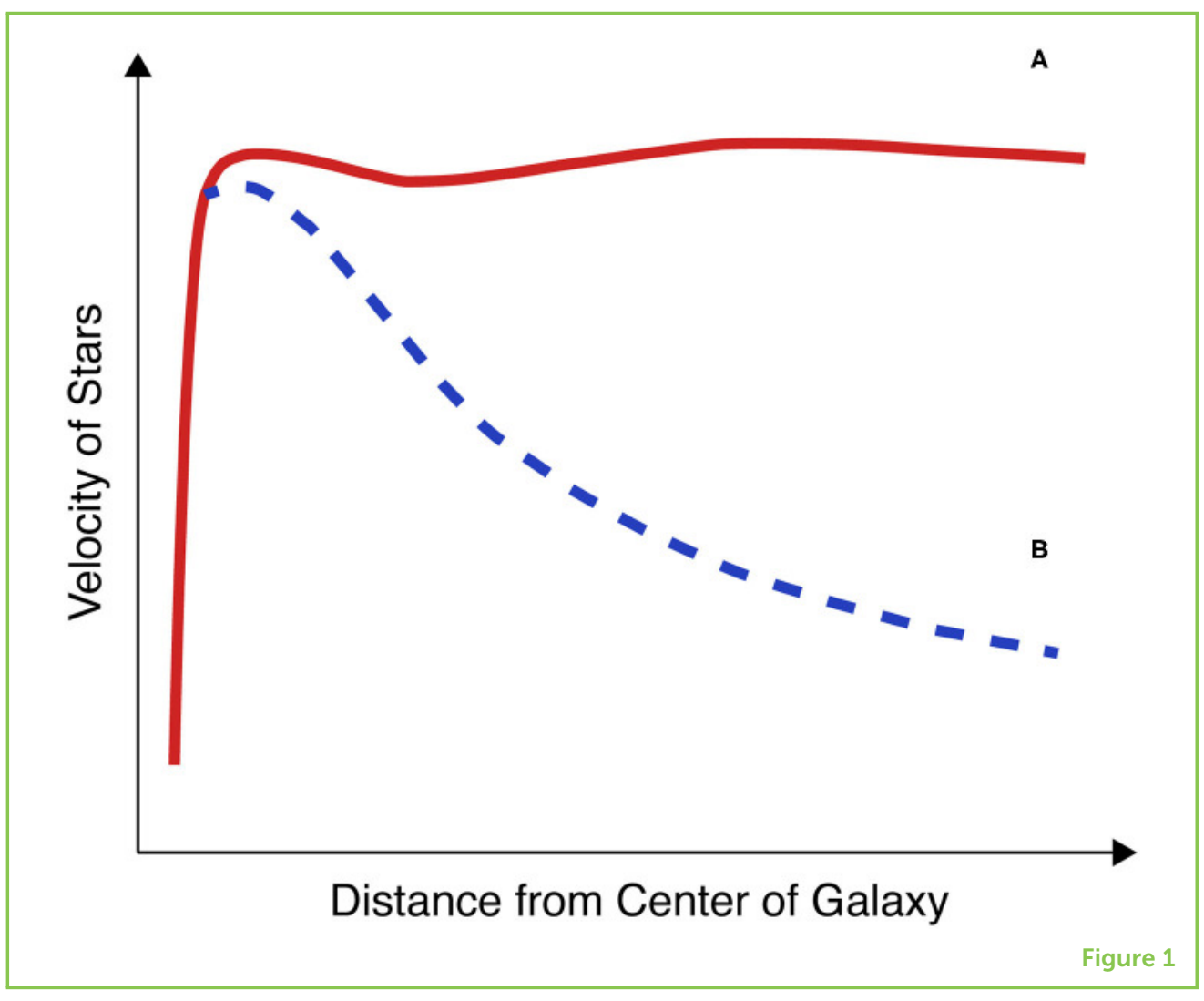

a spiral galaxy) and plotted the velocities of the stars vs. their distances from the center of the galaxy. Scientists discovered that the stars were not behaving in the way anticipated. They found that the stars farther away from the center were moving much faster than predicted (Figure 1 ). The only way this is possible is if there is more mass in the outer parts of galaxies than we can observe. The fact that we are unable to see this mass, because it is not emitting light, suggests the presence of dark matter.

\section{DARK MATTER MESSES WITH CALCULATIONS OF GALAXY MASS}

Evidence for dark matter is not all new. Back in 1933, Fritz Zwicky, a Swiss astronomer, was one of the first to detect the presence of dark matter. Zwicky studied the light emitted by the more than 1,000 galaxies that are part of the Coma Cluster of galaxies. Zwicky determined the mass of the Coma Cluster using two methods. One method used the velocities of the galaxies, which he determined by measuring shifts in the light they emitted. The second calculation method determined mass using the total brightness of the cluster. In comparing the two resulting mass estimates, he found that the galaxy velocity measurement estimated that there is hundreds of time more mass in the Coma Cluster than the brightness estimate predicted. 
Figure 2

An image of the Bullet Cluster produced by NASA's Hubble Space Telescope and the Chandra X-ray Observatory. The Bullet Cluster is a combination of two galaxies that once collided. The blue areas represent the majority of the mass in the galaxies, which is made up of dark matter, and the pink areas are normal matter. We can detect the dark matter through gravitational lensing, which detects shifts in light produced by distant celestial objects [5]. The bright spots outside the colored areas are stars and galaxies that are not part of the Bullet Cluster (Credit: X-ray: NASA/CXC/CfA/ M.Markevitch et al.; Optical: NASA/STScl; Magellan/U.Arizona/ D.Clowe et al.; Lensing Map: NASA/STScl; ESO WFI; Magellan/

U.Arizona/ D.Clowe et al.).

\section{GRAVITATIONAL}

\section{LENSING}

Light produced by distant galaxies bends and distorts as it interacts with the gravitational field of huge amounts of mass such as clusters of galaxies.

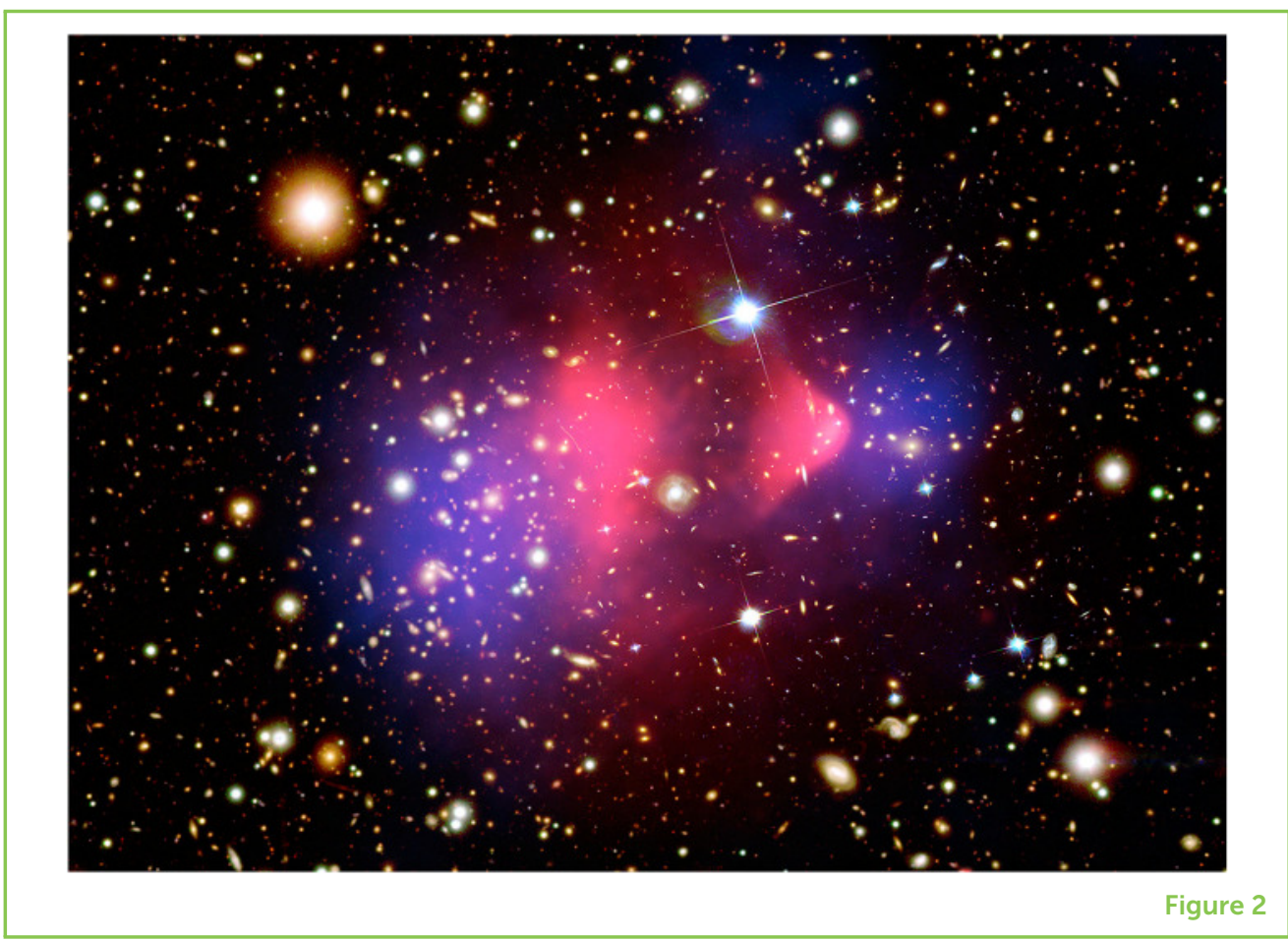

Since the extra matter was not emitting light he said, "If this would be confirmed, we would get the surprising result that dark matter is present in much greater amount than luminous matter" [3]. Very soon after, a similar result was obtained from the Virgo cluster of galaxies. However, measuring techniques at the time were not as precise as modern methods and the controversial nature of the result-that the universe is dominated by some kind of unknown dark matter-caused scientists to reject this hypothesis until almost 50 years later.

\section{DARK MATTER BENDS LIGHT}

The third area of evidence supporting the existence of dark matter comes from a study of the Bullet Cluster, which is the name given to two galaxies that recently collided. Astronomers have found a way to discover the mass of a celestial object, like a galaxy, using a technique known as gravitational lensing [4]. Gravitational lensing is based on the fact that the mass of an object influences the density of space around it. When light travels through this dense space, it bends. To make this clear, let us imagine a flat stretched sheet. The sheet represents space when no masses are near it. Now, imagine placing a bowling ball on the sheet. We know that the sheet will get pulled down by the bowling ball. The ball would curve the sheet in a similar manner to how masses curve spacetime. When light passes near an object in space, it travels on the curved surface, which bends the light waves. The larger the mass of the object, the more the light bends. With the help of this theory, we can determine the mass of a celestial object by watching how much the light from a star right behind it bends. 
Figure 3

The entire circle represents all the matter that was present 380,000 years after the birth of the universe. You can see that the amount of dark matter is much greater than all other kinds of matter. The amount of dark matter remains similar today [2], and the mass of the universe has not changed much since the era shown in the figure. Chart created by NASA/WMAP Science

Team is Public Domain

\section{NEUTRINOS}

Tiny particles, smaller than atoms, that do not have an electrical charge. Neutrinos are one of the components of dark matter.

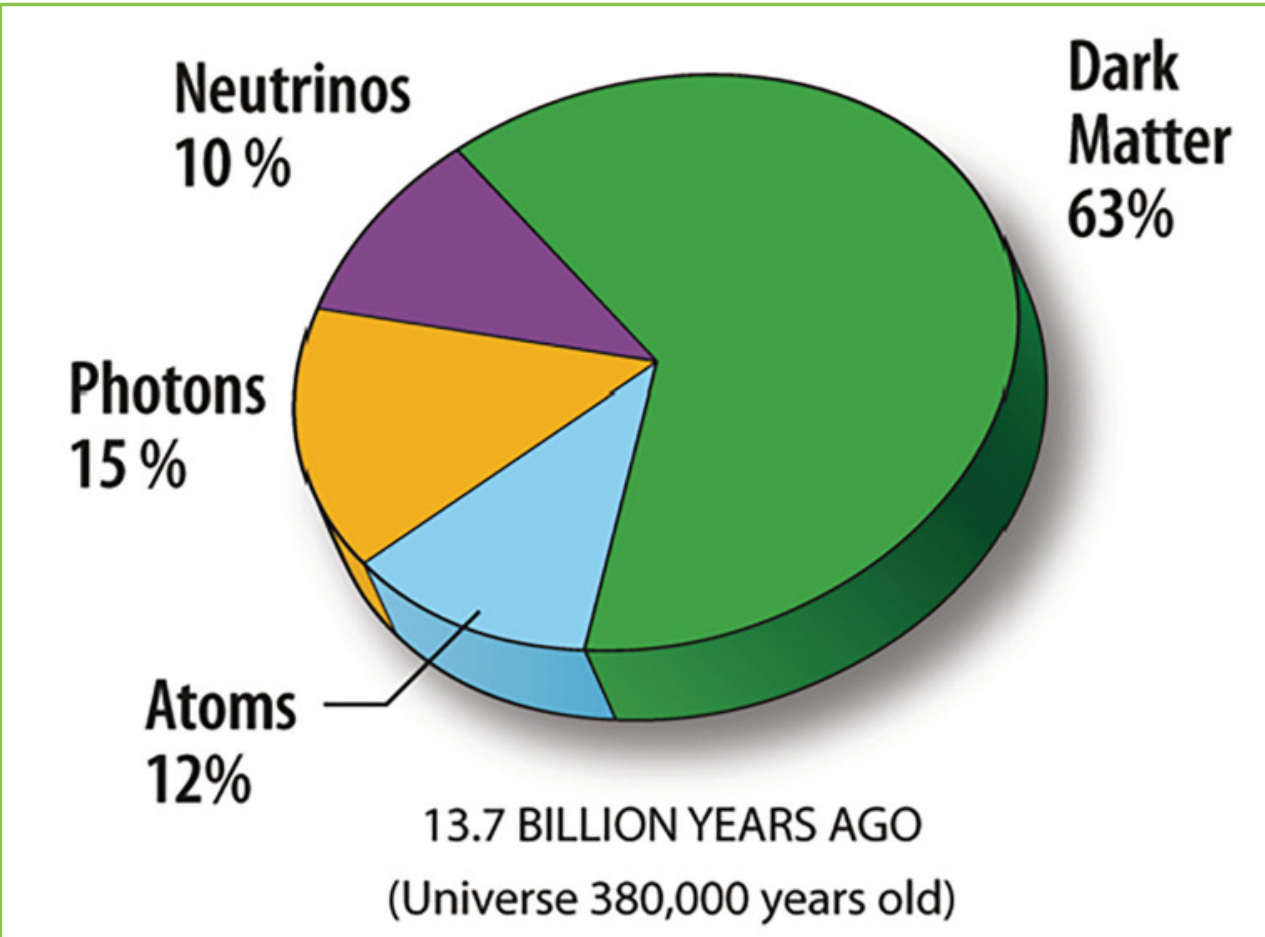

Figure 3

Using gravitational lensing, scientists determined the total mass of the Bullet Cluster, including the dark matter [5]. Figure 2 shows that most of the mass of the Bullet cluster is not located where the source of the $\mathrm{X}$-ray emissions are originating-meaning it is not from the matter we can see. Hence, these galaxies are composed of much more dark matter than regular matter.

\section{WHAT COULD DARK MATTER BE?}

Scientists have proposed many different theories to attempt to solve the dark matter puzzle. Some scientists believe that dark matter is just regular matter that is concentrated in difficult-to-detect objects, such as large planets or black holes. However, scientific observations make this theory unlikely.

So, what is dark matter made of, then? Scientists have already discovered one type of particle that makes up dark matter, called neutrinos. Neutrinos are particles that do not emit light, just like dark matter. However, neutrinos can make up only a fraction of the total amount of dark matter, because they are too light and, when they were created in the early universe, they were moving too fast. So, other yet-to-be-discovered particles must be involved. Two of the most promising candidates are proposed particles called WIMPS and axions. Neither type has been observed yet, and many experiments around the world are currently searching for them. 
1 For information on the LHC, see https://home.cern/ about and on dark matter https://home.cern/ science/physics/darkmatter.

\section{CONCLUSION}

Dark matter makes up about $63 \%$ of all matter in the universe (Figure 3). Indeed, our ability to understand dark matter will help us learn more about the universe, including details of the universe's origin and formation. Many experiments are being carried out around the world, including experiments at the Large Hadron Collider in Switzerland, ${ }^{1}$ to determine the nature of the tiny particles that can tell us about the conditions in which dark matter is formed. Far more work needs to be done, but one thing is for certain-there is a lot to look forward to in the fields of astrophysics and particle physics!

\section{REFERENCES}

1. Garner, R. 2015. About the Hubble Space Telescope. Retrieved from: https:// www.nasa.gov/mission_pages/hubble/story/index.html

2. Ibarra, A. 2015. Dark matter theory. Nucl. Part Phys. Proc. 267-269:323-31. doi: 10.1016/j.nuclphysbps.2015.10.126

3. Bertone, G., and Hooper, D. 2018. History of dark matter. Rev. Mod. Phys. 90:045002. doi: 10.1103/revmodphys.90.045002

4. Wambsganss, J. 1998. Gravitational lensing in astronomy. Living Rev. Relativ. 1:12. doi: 10.12942//rr-1998-12

5. Clowe, D., Bradač, M., Gonzalex, A. H., Markevitch, M., Randall, S. W., Jones, C., et al. 2006. A direct empirical proof of the existence of dark matter. Astrophys. J. Lett. 648:L109-13. doi: 10.1086/508162

SUBMITTED: 25 June 2020; ACCEPTED: 31 March 2021; PUBLISHED ONLINE: 04 May 2021.

EDITED BY: Joey Shapiro Key, University of Washington Bothell, United States

CITATION: Bhathe VP, Brennan C, Ellis S, Moynes E, Graham K and Landsman SJ (2021) How Do Scientists Know Dark Matter Exists? Front. Young Minds 9:576034. doi: 10.3389/frym.2021.576034

CONFLICT OF INTEREST: The authors declare that the research was conducted in the absence of any commercial or financial relationships that could be construed as a potential conflict of interest.

COPYRIGHT (c) 2021 Bhathe, Brennan, Ellis, Moynes, Graham and Landsman. This is an open-access article distributed under the terms of the Creative Commons Attribution License (CC BY). The use, distribution or reproduction in other forums is permitted, provided the original author(s) and the copyright owner(s) are credited and that the original publication in this journal is cited, in accordance with accepted academic practice. No use, distribution or reproduction is permitted which does not comply with these terms. 

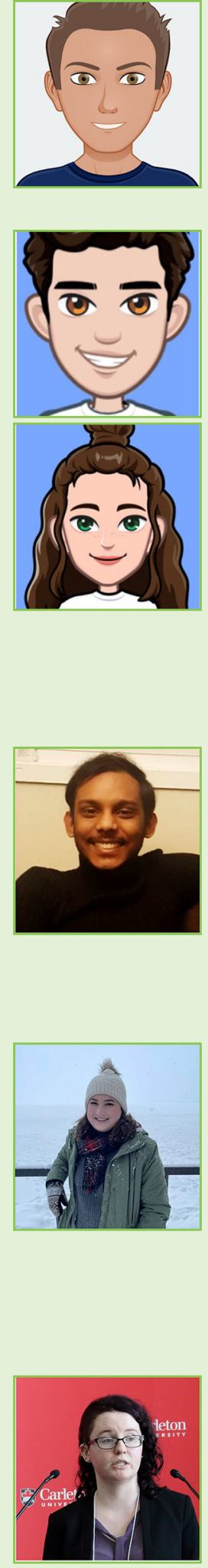

\section{YOUNG REVIEWERS}

NOAH, AGE: 10

Hi! My name is Noah. I am 10 years old, and I just started 5 th grade. Ever since I was 3 years old, I have wanted to become an astronaut. I know a lot about space, aviation, science, and history. I love to read and learn new things. I also like playing with my cousins and friends and participating in a local EAA youth chapter.

\section{OSCAR, AGE: 12}

Hello! My name is Oscar, I live in France, close to Paris. My favorite hobby is playing video games with my friends. I also like hikings and sports, and I like Science a lot. I also like mathematics and physics.

\section{SANAH, AGE: 14}

Hi! My name is Sanah. I live in France, close to Paris. I am very curious about the world, I love Science and especially astronomy, neurosciences, and psychology. When I grow up I want to become a researcher, and help to cure diseases. I also love contemporary dance and music.

\section{AUTHORS}

\section{VISHNU PRITHIV BHATHE}

Vishnu Bhathe recently graduated from Carleton University in Ottawa, Ontario, Canada with a Bachelor of Science degree. Ever since he was a child, he has been curious about science and how people use the lessons from science to better the world. He is also interested in entrepreneurship and aims to use scientific research to fuel innovation. In his free time, he likes to ride bikes, paint, read books, and exploring new things.

\section{CHRISTINA BRENNAN}

Christina Brennan is an undergraduate student at Carleton University in Ottawa, Ontario, Canada majoring in interdisciplinary science. She is especially interested in pursuing a career in science communication, and using the skills developed in her undergrad to bridge the gap between disciplines. Since she was 10 years old, Christina has been designing floor plans of houses on graph paper and continues to do so today through 3D modeling. Aside from her academic goals, she hopes to 1 day design and build her own net zero home.

\section{STEPHANIE ELLIS}

Stephanie is an undergraduate student in her final year of the Interdisciplinary Science and Practice program in Ottawa, Ontario, Canada. She is passionate about community engagement and figuring out how different systems work, from the human brain to computers. She was inspired by her teachers to continue searching for answers and to follow her passions at a young age. 

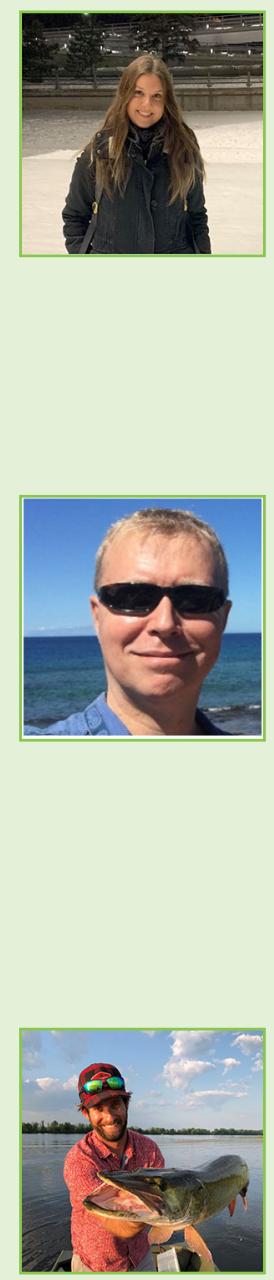

\section{EMILY MOYNES}

Emily is a fifth-year undergraduate student studying Environmental Science at Carleton University in Ottawa, Ontario, Canada. During her schooling, she completed an internship under the supervision of Dr. Steven Cooke on various fish-related projects, resulting in a publication in Transactions of the American Fisheries Society. She is currently completing her honors thesis with Dr. Thomas Sherratt on insect body toughness. She loves acquiring new knowledge and experiences in her field and hopes to 1 day obtain a job exploring animal behavior or working toward conservation-related initiatives.

\section{KEVIN GRAHAM}

Kevin Graham is a professor at Carleton University in Ottawa, Canada specializing in particle physics research. At an early age, Kevin developed an interest in understanding how the universe works at a fundamental level. This led him to become a scientist engaged in measuring the properties and interactions of the smallest particles of matter. Kevin is a member of the ATLAS experiment located on the Large Hadron Collider at CERN near Geneva, Switzerland. This experiment measures the interactions from high-energy proton collisions that could produce dark matter, among other particles. He is working with an international team of scientists to search for evidence of dark matter being created in ATLAS.

\section{SEAN J. LANDSMAN}

Sean is a teaching professor in the Interdisciplinary Science and Practice program at Carleton University in Ottawa, Ontario, Canada on the unceded territory of the Algonquin First Nation. He is a trained fisheries ecologist and studies how fish move about their environments as well as how people affect them. He is also a passionate science communicator and enjoys sharing his knowledge with anyone that will listen! In fact, it was this love of communicating fisheries science that led him to photography and specifically underwater photography. Sean loves to spend time outdoors, especially fishing and hiking, and enjoys tinkering in his basement making things out of wood. *sean.landsman acarleton.ca 